\title{
The Association Between IncRNA LRRC75A-AS1 and The Clinical Characteristics in Neuroblastoma
}

\author{
Zhenni Wang, Shan Wang*, Changchun Li, Chao Yang, Liang Peng and Jian Sun \\ Department of Pediatric Surgical Oncology, Children's Hospital of Chongqing Medical University, Chongqing, China \\ *Corresponding author: Wang Shan, Department of Children's Hospital of Chongqing Medical University, Chongqing, \\ China
}

\section{ARTICLE INFO}

Received: 幽 May 27, 2020

Published: 幽 June 03, 2020

Citation: Zhenni Wang, Shan Wang, Changchun Li, Chao Yang, Liang Peng, Jian Sun. The Association Between IncRNA LRRC75A-AS1 and The Clinical Characteristics in Neuroblastoma. Biomed J Sci \& Tech Res 28(1)-2020. BJSTR. MS.ID.004597.

Abbreviations: NB: Neuroblastoma; lncRNAs: Long Noncoding RNAs; LDH: Lactate Dehydrogenase; NSE: Neuron-Specific Enolase; ORFs: Open Reading Frames; uFH: Unfavorable Histology; FH: Favorable Histology; NA: Not Amplified; NM: Not Metastasis; PD: Progressive Disease; VMA: Vanilla Mandelic;SNHG29: Small Nucleolar RNA Host Gene 29; TJ: Tight Junction; CRC: Colorectal Carcinoma

\section{ABSTRACT}

Background: Neuroblastoma is the highest mortality rate extracranial soild tumor in childhood. Accumulating evidence indicated that long noncoding RNAs (lncRNAs) are widely expressed in neuroblastoma, and playing an important role in the development and progression.

Methods: RNA sequencing was conducted to identify differentially expressed lncRNAs in four III phase and four IV phase tumor tissues of neuroblastoma. RT-qPCR was carried out to validate the result of sequencing. Clinical information was reviewed to analyze the relationship between IncRNA and clinical characteristics. The public database R2 was used to analyze prognosis.

Result: Differentially expressed lncRNAs were identified. LRRC75A-AS1 was the overexpressed lncRNA in IV phase patients. RT-qPCR was conducted in tumor tissues, confirming the tendency with sequencing. And higher expression of LRRC75A-AS1 was associated with N-MYC ( $<<0.001)$, advanced stage ( $p=0.029)$, Risk group ( $p=0.027)$. Furthermore, LRRC75A-AS1 was correlated with Shimada classification( $p=0.046)$, LDH level $(r=0.390, p=0.003)$, D-Dimer level $(r=0.338, p=0.012)$, and NSE level $(r$ $=0.284, \mathrm{p}=0.05)$. The neuroblastoma dataset shows that patients with overexpressed LRRC75A-AS1 have a worse prognosis than down-expressed.

Conclusion: LRRC75A-AS1 is associated with clinical characteristics of neuroblastoma and may function as a prognostic predictor or a therapeutic target.

Keywords: Biomarker; IncRNA LRRC75A-AS1; Neuroblastoma

\section{Introduction}

Neuroblastoma (NB) is a sympathetic embryonic tumor originating from the neural crest of the embryonic sympathetic nervous system,it is the most common extracranial solid tumor in children which accounts for $7-10 \%$ of all childhood cancer mortality [1-5]. In order to take tailored treatment approaches for neuroblastoma, pediatric cooperative groups introduce risk factors including clinical stage, age, histologic category, grade of tumor differentiation, MYCN status, DNA ploidy, and 11q exception $[6,7]$. High-risk neuroblastoma patients often have unfavorable outcomes, with the 5-year overall survival rate less than 50\% [6]. The application of genetic difference analysis promote accurate stratification has attracted widespread attention. Therefore, it is of great practical significance and theoretical value to explore effective drug targets and better biomarkers for advanced neuroblastoma.
Long noncoding RNAs (lncRNAs) refer to endogenous RNAs that are longer than 200 nucleotides and lack of specific complete open reading frames (ORFs) and the function of protein-coding $[8,9]$.

Thus they were once considered a part of transcriptional noise, but now have been proved as potential key regulators of promoting or maintaining tumorigenesis and the development of cancer, having clinical potential as prognostic biomarkers for targeted therapeutics and interventions in various cancers[4,10]. Several lines of evidence have shown that IncRNAs have been implicated in initiation and progression of neuroblastoma [5,6], and IncRNAbased prognostic biomarkers have been proposed for tumor stratification and predicting survival. Therefore, deep investigating of the roles and mechanisms of IncRNAs in tumorigenesis provides promises in developing new biomarkers and molecular-targeted therapy. Our research aims to identify lncRNA-based biomarkers 
that could be used for prognosis prediction and treatment. The purpose of this study was to explore the relationship between lncRNAs and clinicopathological parameters in neuroblastoma patients, to further explore the lncRNAs that lead to the invasion and metastasis of neuroblastoma. In the first, we conducted RNAsequencing to identify differentially expressed lncRNAs in 4 III stage and 4 IV stage patients' tumor tissues of neuroblastoma, and we identified a IncRNA named LRRC75A-AS1 was upregulated IncRNAs in IV stage neuroblastomas, Futher to explore the relationship between LRRC75A-AS1 and clinical characteristics ,RT-qPCR was carried out to detect 57 cases of neuroblastoma.

\section{Materials and Methods}

\section{Patient}

A total of 57 cases of fresh primary tumor tissues with pathologically diagnosed neuroblastoma were collected in this study approved by the ethics committee of the Children's Hospital of Chongqing Medical University from August 2014 to May 2019. And subjects (or their parents or guardians) have given their written informed consent.

\section{The Inclusion Criteria}

a) Pathologically diagnosed NB.

b) Primary tumor without any treatment.

c) Written informed consent was obtained from the guardians.

\section{The Exclusion Criteria}
a) History of other malignant disease.
b) Recurrent or treated disease.
c) The quality of tissues was unqualified.

Clinical features of these patients at diagnosis including age, gender, tumor size, INSS stage, risk group, MYCN status, tumor biomarkers, and metastasis were retrospectively collected. All fresh tissue specimens were preserved in $-80^{\circ} \mathrm{C}$ until use. R2: Genomics Analysis and Visualization Platform (http://r2.amc.nl) was used to investigate the relationship between IncRNA expression and overall survival with neuroblastoma patients.

\section{Expression Profile Analysis of RAN-Sequencing}

The RAN-sequencing was employed to identify neuroblastomarelated RNAs. After hybridization and washing with samples, 8 samples of extracted RNA of neuroblastoma tumor tissues (4 III stage neuroblastoma and 4 IV stage neuroblastoma) were analyzed.

\section{RNA Extraction and Real Time qRT-PCR Analysis}

Total RNA of specimens was extracted using RNA extraction reagent kit (Bio Teke). RNA concentration and purity were measured by NonoDrop (Thermo Scientific). cDNA was reverse transcribed with the Prime Script RT reagent Kit ((Takara Biotechnology Co., Ltd, China) from $1000 \mathrm{ng}$ of total RNA. The real-time qPCR analyses were carried out using SYBR GREEN Premix ExTaq kit (Danfeng, China) by CFX96 Cycler System. Relative RNA expression was computed by $2^{-\Delta \Delta \mathrm{Ct}}$ method with normalization to human $\beta$-actin. The primers for LRRC75A-AS1 are: F 5'- AGCTCACAGCACACCTGGCTA-3'and R $5^{\prime}$-AGCTGAGGCAGGAGGACCAT- ${ }^{\prime}$, and the primers for $\beta$-actin are: 5'-CCTGGCACCCAGCACAAT- ${ }^{\prime}$ ' and R 5'-GGGCCGGACTCGTCATAC- ${ }^{\prime}$.

\section{Statistical Analysis}

Statistical analyses were conducted by SPSS 23.0 (IBM Corporation. Armonk, NY, USA). Graphical depiction of data was generated by GraphPad Prism.v5.0. (GraphPad Software, Inc., La Jolla, CA). In the statistical analysis, a two-sided $p$ value $<=0.05$ was considered statistically significant. Differentially expressed lncRNAs were identified through fold change as well as $\mathrm{P}$ value calculated with t-test. The threshold set for up- and downregulated genes was a fold change $>=2.0$ and a p value $<=0.05$. For qualitative data, the $\chi 2$ test or Fisher exact test was used to evaluate the significance between groups. For quantitative data, Kruskal-Wallis test was used to analyze the significance between individual groups. The correlations were analyzed by Spearman correlation analysis. The prognostic relationship was evaluated using Kaplan-Meier.

\section{Result}

\section{Screening for Differentially Expressed lncRNAs}

To investigate marrow metastasis-related RNA expression profile in neuroblastoma tissues, we analyzed 8 tissue samples of neuroblastoma (4 III stage and 4 IV stage patients) by using the RNA-sequencing. The pathological characteristics of the 8 patients are listed in Table 1 . With the threshold set for up- and downregulated genes of a fold change $>=2.0$ and a p value $<=0.05$, 1043differentially expressed IncRNAs were identified between III and IV stage tumor samples, including 458 upregulated lncRNAs and 585 downregulated IncRNAs. Among them, we found that LRRC75A-AS1 was the overexpressed IncRNA in IV stage patients with the fold change of $3.19(p=0.02$, (Figure 1). To determine the tendency of sequence, RT-qPCR was carried out to measure LRRC75A-AS1expression level of 57 neuroblastoma tumor tissues, including the 8 patients 'tumor tissues for sequencing. The relative expression of LRRC75A-AS1 ranged from 0.03 to 5.62, with the median value of 0.39 . Compared to the III stage tissues, expression of LRRC75A-AS1was higher in IV stage tissues, however it's not statistically significant ( $\mathrm{p}=0.152$ ). But compared to the early stage tissues, expression of LRRC75A-AS1was higher in advanced stage tissues ( $p=0.029$ ) (Figure 2A). LRRC75A-AS1 was higher in highrisk than intermediate-risk and low-risk neuroblastoma $(p=0.027)$ (Figure 2B). LRRC75A-AS1 was higher in N-MYC amplified disease than N-MYC Not-amplified disease $(p<0.001)$ (Figure $2 C)$. The results suggesting that IncRNA LRRC75A-AS1 may play a significant role in the pathogenesis and development of neuroblastoma. 


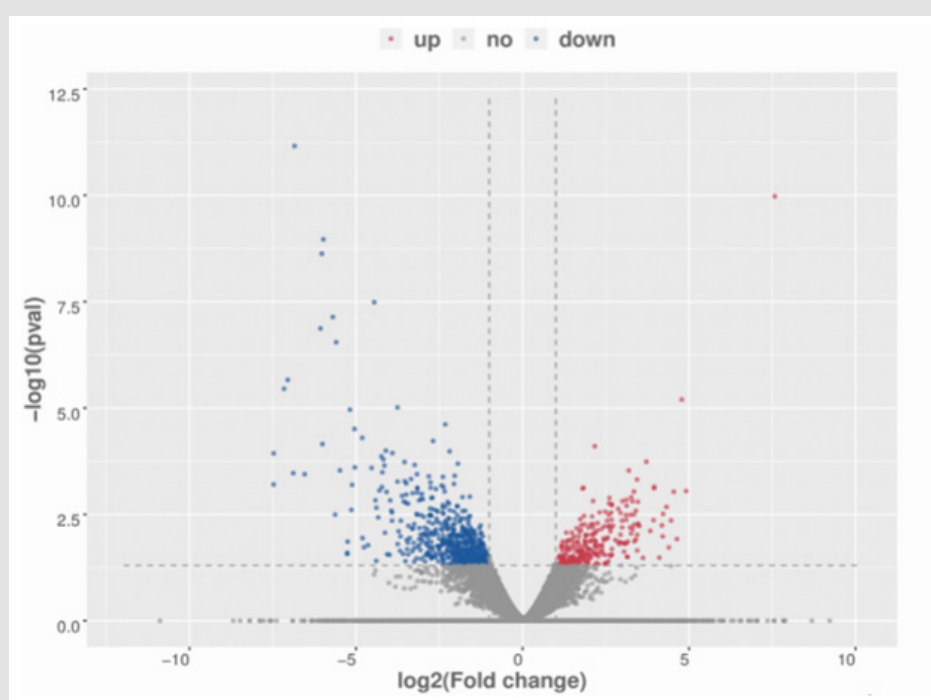

Figure 1: The volcano plot showed differentially expressed lncRNAs between III stage and IV stage neuroblastoma tumor samples.

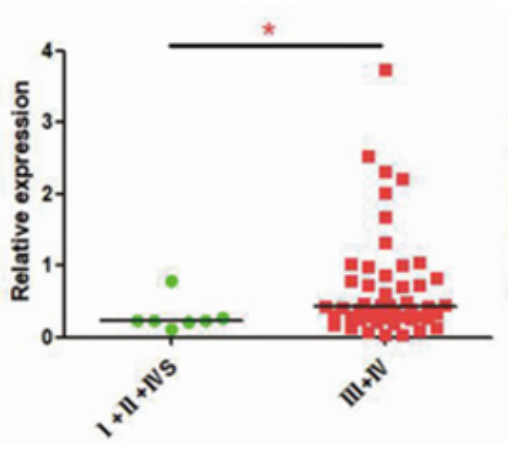

Figure 2(A)

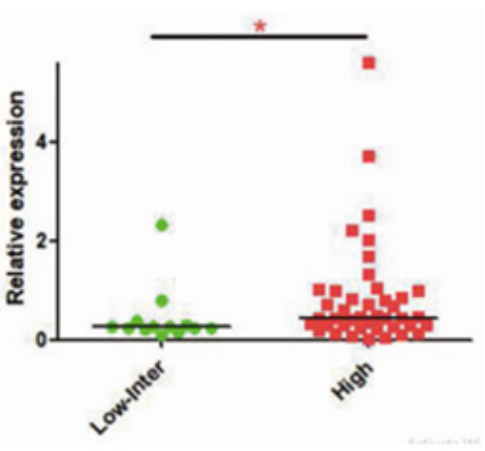

Figure 2(B)

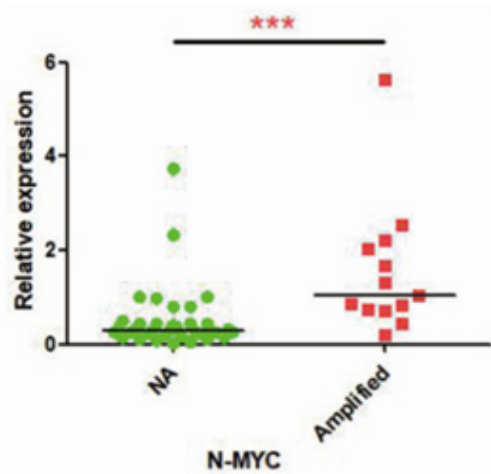

Figure $2(C)$

Figure 2: The correlation between expression of LRRC75A-AS1 and Stage, Risk classification, N-MYC.

(A) Relative expression of LRRC75A-AS1 in early (I, II, IVs) and advanced (III, IV) stage disease.

(B) Relative expression of LRRC75A-AS1 in low and intermediate (low + inter) and high-risk neuroblastoma.

(C) Relative expression of LRRC75A-AS1 in N-MYC(MYCN) not amplified, and N-MYC amplified. 
Table 1: Clinicopathological parameters of 8 patients whose tumors were used for RNA-sequencing.

\begin{tabular}{|c|c|c|c|c|c|c|c|c|}
\hline Patients & Age & Gender & INSS Stage & Risk Group & $\begin{array}{c}\text { Histologic } \\
\text { Classification }\end{array}$ & MYCN Status & $\begin{array}{c}\text { Marrow } \\
\text { Metastasis }\end{array}$ & Outcome \\
\hline A1 & 6 years & Female & IV & High & uFH & NA & Metastasis & Alive \\
\hline A2 & 5 years & Female & IV & High & uFH & NA & Metastasis & - \\
\hline A3 & 4 years & Female & IV & High & uFH & NA & Metastasis & Alive \\
\hline A4 & 6 years & Male & IV & High & uFH & NA & Metastasis & Alive \\
\hline B1 & 4 years & Female & III & High & uFH & NA & NM & PD \\
\hline B2 & 1 years & Male & III & High & uFH & NA & NM & Alive \\
\hline B3 & 3 years & Female & III & High & uFH & NA & NM & Alive \\
\hline B4 & 12 years & Male & III & High & uFH & NA & NM & Alive \\
\hline
\end{tabular}

Note: INSS: International Neuroblastoma Staging System; Inter: Intermediate; uFH: Unfavorable Histology; NA: Not Amplified; NM: Not Metastasis; PD: Progressive Disease

\section{Correlations Between the Expression Level of LRRC75A- AS1 and Clinical Characteristics}

The 57 neuroblastoma patients were divided into two groups (high or low) based on the median value of LRRC75A-AS1 expression level (Table 2). We found that high LRRC75A-AS1 expression level in tumor tissues was associated with advanced INSS stage ( $\mathrm{p}=$ $0.039)$, risk group ( $p=0.004)$, N-MYC status $(p=0.001)$, shimada classification ( $p=0.046$ ). Moreover, we identified that LRRC75AAS1 expression was correlated with serum lactate dehydrogenase (LDH) level ( $\mathrm{r}=0.390, \mathrm{p}=0.003)$ (Figure 3A), D-Dimer level $(\mathrm{r}=$ $0.338, p=0.012$ ) (Figure $3 \mathrm{~B}$ ), and serum neuron-specific enolase (NSE) level $(r=0.284, p=0.05)$ (Figure $3 C$ ) ,but has no correlation with ki-67 level ( $p=0.163)$,Vanilla mandelic acid(VMA) level ( $p=$ 0.073 ) , and tumor size $(\mathrm{p}=0.515)$.

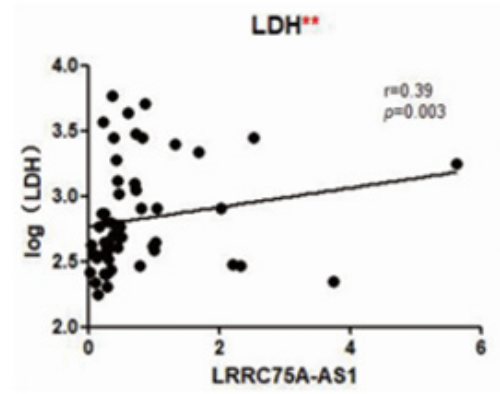

Figure 3(A)
D-Dimer

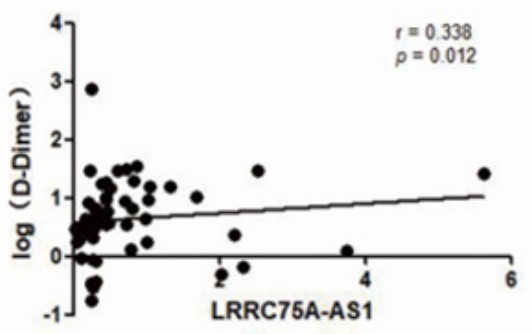

Figure 3(B)

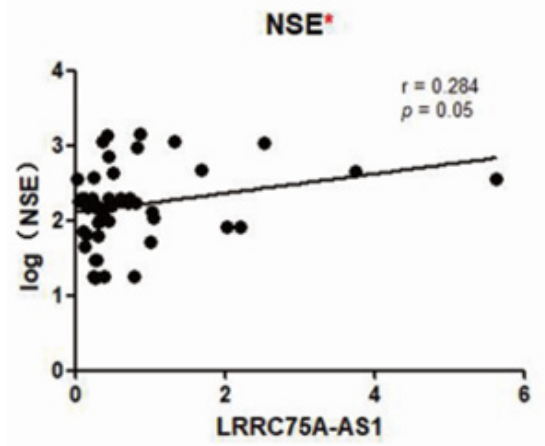

Figure $3(C)$

Figure 3: The correlation between expression of LRRC75A-AS1.

(A) $\mathrm{LDH}$

(B) D-Dimer

(C) NSE 
Table 2: Relationships between the expression of LRRC75A-AS1 and clinicopathological parameters in neuroblastoma

\begin{tabular}{|c|c|c|c|c|}
\hline \multirow{2}{*}{ Factors } & \multicolumn{2}{|c|}{ LRRC75A-AS1 Expression } & \multirow{2}{*}{$\mathrm{x} 2$} & \multirow{2}{*}{$\mathbf{P}$} \\
\hline & $\operatorname{High}(n=29)$ & $\operatorname{Low}(n=28)$ & & \\
\hline \multicolumn{3}{|c|}{ Age } & \multirow{3}{*}{2.354} & \multirow{3}{*}{0.155} \\
\hline$\geq 1$ year & 23 & 17 & & \\
\hline$<1$ year & 6 & 11 & & \\
\hline \multicolumn{3}{|c|}{ Gender } & \multirow{3}{*}{0.422} & \multirow{3}{*}{0.592} \\
\hline Male & 19 & 16 & & \\
\hline Female & 10 & 12 & & \\
\hline \multicolumn{3}{|c|}{ INSS Stage } & \multirow{3}{*}{4.275} & \multirow{3}{*}{0.039} \\
\hline $\mathrm{I}+\mathrm{II}+\mathrm{IVS}$ & 1 & 6 & & \\
\hline III +IV & 28 & 22 & & \\
\hline \multicolumn{3}{|l|}{ Risk group } & \multirow{3}{*}{8.489} & \multirow{3}{*}{0.004} \\
\hline Low +Intermediate & 2 & 11 & & \\
\hline High & 27 & 17 & & \\
\hline \multicolumn{3}{|c|}{ Shimada Classification } & \multirow{4}{*}{3.987} & \multirow{4}{*}{0.046} \\
\hline $\mathrm{uFH}$ & 25 & 15 & & \\
\hline $\mathrm{FH}$ & 4 & 9 & & \\
\hline Missing & 0 & 4 & & \\
\hline \multicolumn{3}{|c|}{ N-MYC Status } & \multirow{4}{*}{11.924} & \multirow{4}{*}{0.001} \\
\hline Amplified & 12 & 1 & & \\
\hline NA & 14 & 24 & & \\
\hline Missing & 3 & 3 & & \\
\hline \multicolumn{3}{|c|}{ Marrow Status } & \multirow{3}{*}{2.998} & \multirow{3}{*}{0.083} \\
\hline Metastasis & 17 & 10 & & \\
\hline NM & 12 & 18 & & \\
\hline
\end{tabular}

Note: INSS: International Neuroblastoma Staging System; uFH: Unfavorable Histology; FH: Favorable Histology; NA: Not Amplified; NM: Not Metastasis Relations Between Expression Level of LRRC75A-AS1
and Prognosis in Neuroblastoma Patients

Owing to the small sample size and limited follow-up time of partial patients, we cannot analyze the correlation between overall survival and LRRC75A-AS1 in our patients. Thence, we see progressive disease or died as outcome indicator to investigate the relationship of LRRC75A-AS1 expression level and prognosis with neuroblastoma patients. The results show that high expression of LRRC75A-AS1 was correlated with poor prognosis $(\chi 2=5.662$, $p=0.017$ ) (Table 3). Further to investigate the relationship of

Table 3: The relationship of LRRC75A-AS1 expression and overall survival.

\begin{tabular}{|c|c|c|c|c|}
\hline \multirow{2}{*}{ Outcome } & \multicolumn{2}{|c|}{ LRRC75A } & \multirow{2}{*}{$\chi^{2}$} & \multirow{2}{*}{$\mathbf{p}$} \\
\hline & High & Low & & \\
\hline PD/Died & 14 & 6 & \multirow{3}{*}{5.662} & \multirow{3}{*}{0.017} \\
\hline Live & 9 & 17 & & \\
\hline Missing & 6 & 5 & & \\
\hline
\end{tabular}

LRRC75A-AS1 expression and overall survival in neuroblastoma patients. R2: Genomics Analysis and Visualization Platform (http://r2.amc.nl) was used to analyze the relationship (Figure 4). Kaplan-Meier analysis demonstrated elevated LRRC75A-AS1 expression levels were associated with poor prognosis, whereas low expression of LRRC75A-AS1 was associated with favorable outcome in the Versteeg dataset consisting of a cohort of 88 neuroblastoma patients $(n=88$, log-rank: $p=0.027)$. To sum up, our analysis of LRRC75A-AS1 with clinical features and microarray dataset indicated that LRRC75A-AS1 was a novel prognostic marker in neuroblastoma. 


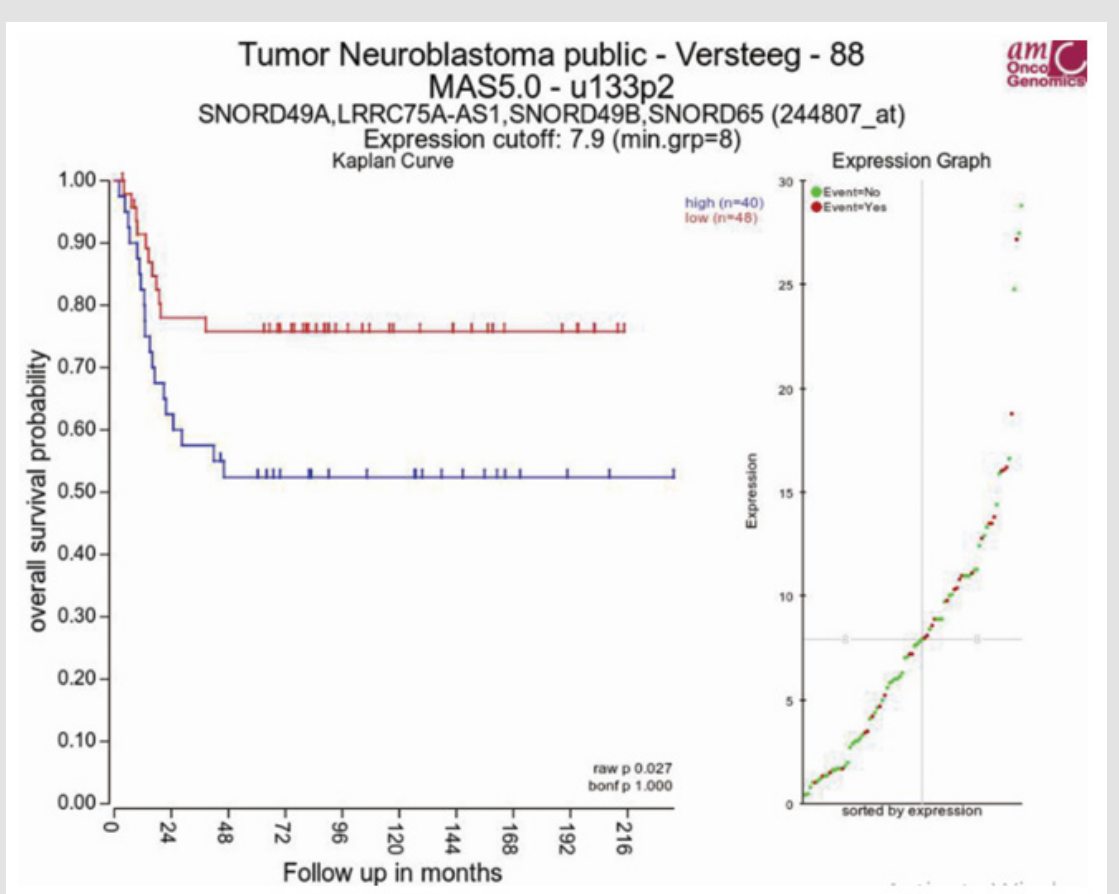

Figure 4: The relationship of LRRC75A-AS1 expression and overall survival in neuroblastoma patients. Kaplan-Meier survival plot was downloaded from R2 Genomics Analysis and Visualization Platform (+).

\section{Discussion}

Over the last decade, with the extensive development of genomic transcription study, accumulative studies indicated that lncRNA have risen to prominence with important roles in a broad range of biological processes. Recent studies have reported that several IncRNAs, for example, MALATA, CASC15, LOC440896, LINC00632, IGF2-AS have been implicated in characteristics and prognosis of neuroblastoma $[5,6,11]$. Therefore, IncRNA have the potential to serve as novel biomarkers for neuroblastoma diagnosis or prognosis. However, the biological functions of most lncRNAs have yet to be explored. High-risk neuroblastoma patients often have unfavorable outcomes, it is one of the biggest obstacles to improve overall survival of neuroblastoma. Hence, it is urgent to investigate the novel genes and illustrate the molecular mechanisms of neuroblastoma. The main purpose of this study was to investigate the differential expression of LRRC75A-AS1 in neuroblastoma, and to find new prognostic and diagnostic markers for neuroblastoma. The results of this study show that LRRC75A-AS1 is up-regulated in neuroblastoma.

Small nucleolar RNA host gene 29 (SNHG29) was also known as LRRC75A-AS1, TSAP19, C17orf45, NCRNA00188, FAM211AAS1, C17or f76-AS1, it's a long noncoding RNA that leucine rich repeat containing 75 A-antisense RNA1 is located on 17p11.2 [12]. Emerging reports has revealed that LRRC75A-AS1 was involved in several biological processes through modulation of signaling pathway, Jeong et al. [13] reported that LRRC75A-AS1 can regulate the vascular calcification negatively, and might act as a possible target in the treatment of vascular calcification. Wang et al. [14] found that LRRC75A-AS1 can regulate the expression of tight junction (TJ) proteins through LRRC75A, affecting the inflammatory responses of bovine mammary epithelial cells. Leavey $\mathrm{K}$ et al. [15] show that LRRC75A is abnormally expressed in the process of normal villous maturation.

In cancers, LRRC75A-AS1 has been served as a crucial regulator in a variety types of cancers including osteosarcoma $[12,16]$, colorectal carcinoma [17], breast cancer [18], gastric cancer [19], glioblastoma [20] and acute myeloid leukemia [21]. Joeri Both et al. $[12,15]$ claimed that LRRC75A serves as malignant facilitatorin in osteosarcoma. Jianxiong Chen et al. [16] reported that LRRC75AAS1 inhibits cell proliferation and migration in colorectal carcinoma, and it might serve as an anti-oncogene for colorectal carcinoma (CRC) tumorigenesis and advancement. Lizhang Han et al. [20] found that SNHG2(LRRC75A-AS1) can regulates miR-223$3 p / C T N N D 1$ axis to promote glioblastoma progression via Wnt/ $\beta$ catenin signaling pathway. FANGCE WANG et al. [21] proved that LRRC75A-AS1 can significantly predict prognosis of acute myeloid leukemia. These papers showed that LRRC75A-AS1 may become a novel molecular marker for diagnosis and treatment of cancer. However, little information of the prognostic value and the role of LRRC75A-AS1 in neuroblastoma has been reported.

In the present study, we compare the expression profile of IncRNA between 4 III phase and $4 \mathrm{IV}$ phase tumor tissues of neuroblastoma by using the RNA-sequencing. We found that LRRC75A-AS1 was the overexpressed IncRNA in IV stage patients with the fold change of 3.19. To further confirm the relationship of LRRC75A-AS1 in neuroblastoma, the RT-qPCR analysis was used 
to analyze the clinical tissue from neuroblastoma patients. These experimental results showed that LRRC75A-AS1was obviously high in advanced stage neuroblastoma, and high expression level of LRRC75A-AS1 was associated with advanced stage disease, high risk group, N-MYC Amplified, unfavorable histology, and the level of LDH, D-Dimer and NSE, which are strong predictors for prognosis of neuroblastoma. Furthermore, we used public neuroblastoma dataset in R2 validated that overexpression of LRRC75A-AS1 was correlated with unfavorable prognosis in neuroblastoma. But, the expression of LRRC75A-AS1 was not correlated with ki-67 level Vanilla mandelic acid(VMA) level and tumor size, the inconsistency may be caused by the small sample size of this study which did not permit attainment of statistical significance.

The limitation of current experiments is the small sample size resulting in limited statistical power, hence, it is still necessary to expand the clinical sample size and patients should also be long-term followed up to validate the prognosis in the public neuroblastoma dataset in our cohort. This study merely proved the relationship between LRRC75A-AS1 and tumor in clinic, so more relevant basic experiments should be practiced on animals and cells. Further studies are needed to illuminate the underlying molecular mechanisms that LRRC75A-AS1 might promote the tumorigenesis and progression of NB, as well as screening for potential therapeutic target for neuroblastoma.

\section{Conclusion}

In conclusion, our study demonstrated that LRRC75A-AS1 was up-regulated in IV phase tumor. Further experiments revealed that overexpression of LRRC75A-AS1 in tumor tissues was associated with aggressive disease including INSS III, IV stage, high risk group, N-MYC amplified, uFH classification, high level of LDH, D-Dimer and NSE, and unfavorable overall survival. Thus LRRC75A-AS1 may function as a potential prognostic biomarker in neuroblastoma, and we conjecture a novel prognostic model including LRRC75AAS1 may predict the outcomes of neuroblastoma patient in clinical practice more accurately.

\section{Statements}

\section{Acknowledgement}

We are grateful for the help from all participators. This work was supported by the General project of clinical medicine research of Children's Hospital Affiliated to Chongqing Medical University (No. YBXM-2019-003) and Chongqing Science and Technology Bureau Project (No. cstc2019jscx-msxmX0220 and cstc2016shmsztzx0042)

\section{Statement of Ethics}

This research complies with the guidelines for human studies. This study approved by the ethics committee of the Children's Hospital of Chongqing Medical University and subjects (or their parents or guardians) have given their written informed consent.

\section{Disclosure Statement}

The authors have no conflicts of interest to declare

\section{Funding Sources}

This work was supported by grants from the General project of clinical medicine research of Children's Hospital Affiliated to Chongqing Medical University (No.YBXM-2019-003) received by W.S., Chongqing Science and Technology Bureau Project (No. cstc2019jscx-msxmX0220 and cstc2016shms-ztzx0042) received by W.S. No funding bodies had any role in study design, data collection and analysis, decision to publish, or preparation of the manuscript.

\section{Author Contributions}

WZ and WS designed the experiments, analyzed the data and revised the manuscript. WZ wrote the manuscript. WZ performed most of the experiments. LC, YC, PL and SJ collected tumor tissues and performed the experiments. All of the authors discussed the results and reviewed the manuscript.

\section{References}

1. Saunders WB (2012) Seminars in pediatric surgery [J] 21(1): 2-14.

2. Maris JM (2010) Recent Advances in Neuroblastoma[J]. N Engl J Med 362(23): 2202-2211.

3. Westermann F, Schwab M (2002) Genetic parameters of neuroblastomas[J]. Cancer Letters 184(2): 127-147.

4. Lin C, Yang L (2018) Long noncoding RNA in cancer: wiring signaling circuitry[J]. Trends Cell Biol 28: 287-301.

5. Irwin MS, Park JR (2015) Neuroblastoma Paradigm for Precision Medicine[J]. Pediatric Clinics of North America 62(1): 225-256.

6. Pinto NR, Applebaum MA, Volchenboum SL, Katherine K Matthay, Wendy B London, et al. (2015) Advances in risk classifification and treatment strategies for neuroblastoma[J]. Clin Oncol 33(27): 3008-3017.

7. Maris JM (2010) Recent advances in neuroblastoma[J]. N Engl J Med 362(23): 2202-2211

8. Tsai MC, Spitale RC, Chang HY (2011) Long intergenic noncoding RNAs: new links in cancer progression[J]. Cancer Res 71: 3-7.

9. Deniz E, Erman B (2017) Long noncoding RNA (lncRNA), a new paradigm in gene expression contro[J]l. Funct Integr Genomics 17: 135-143.

10. Arnes L, Liu Z, Wang J, Carlo Maurer, Irina Sagalovskiy, et al. (2019) Comprehensive characterisation of compartment-specific long noncoding RNAs associated with pancreatic ductal adenocarcinoma[J]. Gut: gutjinl-2017-314353.

11. Srinivasulu, Yerukala, Sathipati, Yenching Lin, Shinn-Ying Ho (2019) Identification and characterization of the IncRNA signature associated with overall survival in patients with neuroblastoma[J]. Scientific reports 9(1): 5125 .

12. Both J, Wu T, Ten Asbroek AL, Baas F, Hulsebos TJ (2016) Oncogenic properties of candidate oncogenes in chromosome region $17 \mathrm{p} 11.2 \mathrm{p} 12$ in human osteosarcoma[J]. Cytogenet Genome Res 150: 52-59.

13. Jeong G, Kwon DH, Shin S, Choe N, Ryu J, et al. (2019) Long noncoding RNAs in vascular smooth muscle cells regulate vascular calcifification[J]. Sci Rep 9: 5848.

14. Wang X, Wang H, Ming-Qing Gao (2020) LRRC75A antisense lncRNA1 knockout attenuates inflammatory responses of bovine mammary epithelial cells[J]. Int J Biol Sci 16(2): 251-263. 
15. Leavey K, Benton SJ, Grynspan D, Bainbridge SA, Morgen EK, et al. (2017) Gene markers of normal villous maturation and their expression in placentas with maturational pathology[J]. Placenta 58: 52-59.

16. Both J, Wu T, Bras J, Gerard R. Schaap, Frank Baas, et al. (2012) Identification of Novel Candidate Oncogenes in Chromosome Region 17p11.2-p12 in Human Osteosarcoma[J]. PLOS ONE 7(1): e30907.

17. Chen J, Lan J, Zhiwei Ye, Shiyu Duan, Yukun Hu, et al. (2019) Long noncoding RNA LRRC75A-AS1 inhibits cell proliferation and migration in colorectal carcinoma[J]. Exp Biol Med (Maywood) 244(14): 11371143.

18. Lv M, Xu P, Wu Y, Huang L, Li W, et al. (2016) LncRNAs as new biomarkers to differentiate triple negative breast cancer from non-triple negative breast cancer[J]. Oncotarget 7: 13047-13059.

ISSN: 2574-1241

DOI: $10.26717 /$ BJSTR.2020.28.004597

Shan Wang. Biomed J Sci \& Tech Res

(c) (P) This work is licensed under Creative

Submission Link: https://biomedres.us/submit-manuscript.php
19. Cao WJ, Wu HL, He BS, Zhang YS, Zhang ZY (2013) Analysis of long noncoding RNA expression profifiles in gastric cancer[J]. World J Gastroenterol 19: 3658-3664.

20. Han L, Li Z, Jiang Y, Zheng Jiang, Ling Tang (2019) SNHG29 regulates miR-223-3p/CTNND1 axis to promote glioblastoma progression via Wnt/ $\beta$-catenin signaling pathway[J]. Cancer Cell Int 19: 345.

21. Wang, Tian, Zhou, Guangming Wang, Wenlei Yu, et al. (2018) A threelncRNA signature for prognosis prediction of acute myeloid leukemia in patients[J]. Molecular Medicine Reports 18(2): 1473-1484.

\begin{tabular}{lll}
\hline & Assets of Publishing with us \\
\hline RIOMEDICAL & Global archiving of articles \\
\hline & - Immediate, unrestricted online access \\
\hline
\end{tabular}

